\title{
HOT DEFORMATION BEHAVIOR OF SUPERALLOY 718
}

\author{
C.I. Garcia, G.D. Wang*, D.E. Camus, E.A. Loria** and A.J. DeArdo \\ Basic Metals Processing Research Institute \\ Department of Materials Science and Engineering \\ University of Pittsburgh \\ * Department of Metal Forming \\ Northeastern University \\ Shenyeng, Liaoning 11006, China \\ ** Metallurgical Consultant
}

\begin{abstract}
The hot deformation behavior of Alloy 718 was characterized on the basis of the dynamic materials model and compression data in the temperature range of 900 to $1177^{\circ} \mathrm{C}$ and strain rate range of 0.005 to $5 \mathrm{~s}^{-1}$. The flow curves at all temperatures and strain rates showed little dependence on strains larger than 0.3. Constitutive equations were used to characterize the dependence of flow stress on strain, strain rate and temperature. A threedimensional distribution of strain rate sensitivity with strain rate and temperature revealed a decrease in sensitivity with an increase in strain rate and a decrease in temperature. A processing (power dissipation) map constructed from the dynamics materials model and the corresponding isoefficient contours at a strain of 0.5 cxposed a domain of peak efficiency of $35 \%$ at temperatures of 1132 to $1177^{\circ} \mathrm{C}$ and the strain rates of 0.05 to $0.5 \mathrm{sec}^{-1}$, which would be optimum parameters for hot working. These results are in good agrecment with previous recrystallization-temperature-time maps reported in the literature. The activation energy for plastic flow in the 900 to $1177^{\circ} \mathrm{C}$ range was about $483 \mathrm{KJ} / \mathrm{mole}$, and the constitutive relationship between flow stress and temperature compensated strain rate (ZenerHollomon parameter) was found to be valid in the temperature range of 900 to $1177^{\circ} \mathrm{C}$.
\end{abstract}

\section{INTRODUCTION}

In order to produce superior feed stock for the forging of turbine disks and other components, attention must be directed to the conditions which provide microstructural grain refinement in Alloy 718 through proper thermomechanical processing during ingot-to-billet conversion. The approach taken in our initial paper ${ }^{(1)}$ was to simulate the commercial operation on forging a $28 \mathrm{in}$. diameter VAR ingot into a $10 \mathrm{in}$. diameter billet via compression testing of specimens taken from that ingot. The results revealed that, under certain TMP conditions, substantial refinement can be obtained and the final microstructure was uniform. Since the microstructure which occurs after a given homogenization treatment and continuous cooling to the deformation temperature is an important factor in the ingot-tobillet conversion process, our second paper ${ }^{(2)}$ provided continuous cooling transformation diagrams and defined a temperature-deformation-recrystallization map for this alloy. As a continuation of the study, the present work provides an analysis of flow stress data in terms of strain rate sensitivity and temperature sensitivity as important hot deformation parameters, and it develops a processing (power dissipation) map on the basis of the principles of the dynamic materials model ${ }^{(3-5)}$. Also, the apparent activation energy was calculated and the

Superalloys 718, 625, 706 and Various Derivatives

Edited by E.A. Loria

The Minerals, Metals \& Materials Society, 1994 
constitutive relationship between flow stress and temperature compensated strain rate (ZenerHollomon parameter) was determined.

\section{EXPERIMENTAL PROCEDURE}

An as-cast workpiece of Alloy 718 was cut into bars with section $2.3 \mathrm{~cm} . \times 2.54 \mathrm{~cm}$. which were homogenized at $1190^{\circ} \mathrm{C}$ for $24 \mathrm{hr}$. After homogenization, the bars were water quenched to room temperature, and machined into cylindrical samples with dimensions 1.14 $\mathrm{cm}$. diameter $\times 1.71 \mathrm{~cm}$. height.

The hot deformation testing was performed using a computer-controlled MTS machine dedicated to high temperature deformation studies that has been previously described ${ }^{(1)}$. The true stress-true strain curves for strain rates $5,0.5,0.05,0.005 \mathrm{~s}^{-1}$ and deformation temperatures $1173,1227,1283,1339,1394$, and $1450 \mathrm{~K}(900,954,1010,1066,1121$ and $1177^{\circ} \mathrm{C}$ ) were obtained.

\section{RESULTS AND DISCUSSION}

The understanding of the microstructural evolution of a given material prior to and after thermomechanical processing requires the development of physical models. This information in turn can be used to describe the thermomechanical condition of the material using well known constitutive equations. This paper attempts to describe the hot deformation behavior of alloy 718 using the above approach.

\section{General Expression of Constitutive Equation} as:

In high temperature deformation, the constitutive equation can be generally expressed

$$
\sigma=\sigma(\dot{\epsilon}, \mathrm{T}, \mathrm{S})
$$

where $\quad \dot{\epsilon}-\quad$ plastic strain rate

$\mathrm{T}$-- deformation temperature (absolute)

S -- parameter which represents the microstructural characteristics of the material, and hence, controls its deformation behavior.

Equation 1, can also be written in terms of the total true strain $(\epsilon)^{(6)}$,

$$
\sigma=\sigma(\dot{\epsilon}, \mathrm{T}, \epsilon)
$$

The flow stress behavior under a given set of strain rate and temperature conditions is usually written as:

$$
\dot{\epsilon}=\operatorname{Af}(\sigma) \mathrm{g}(\mathrm{T})
$$

where, A is a structure dependent constant, $f(\sigma)$ varies with the stress level, and $g(T)$ is typically given by:

$$
\mathrm{g}(\mathrm{T})=\exp (-\mathrm{Q} / \mathrm{RT})
$$

where, $\mathrm{Q}$ is a apparent activation energy for the deformation process. $\mathrm{R}$ and $\mathrm{T}$ have their usual meaning. An example of the typical true stress-true strain curves obtained in this study 
are illustrated in Figure 1. These curves were obtained for different combinations of strain rates and temperatures. As expected, the results illustrated in Figure 1 show the dependence of flow stress with strain rate and deformation temperature. Hence, the true stress as function of strain rate can be calculated using a polynomial equation of $\log (\sigma)$ vs $\log (\dot{\epsilon})$ for a given deformation temperature:

$$
\log (\sigma)=\mathrm{a}+\mathrm{blog} \dot{\epsilon}+\mathrm{c}(\log \dot{\epsilon})^{2}
$$

the value of the constants $\mathrm{a}, \mathrm{b}$ and $\mathrm{c}$ at different temperatures is shown in Table 1.

Table 1. Constants in Equation (5)

\begin{tabular}{|c|c|c|c|}
\hline \hline Temperature, $^{\circ} \mathbf{K}$ & $\mathbf{a}$ & $\mathbf{b}$ & $\mathbf{c}$ \\
\hline \hline 1172 & 2.5977 & 0.0342 & -0.02126 \\
\hline 1227 & 2.5816 & 0.0885 & -0.02364 \\
\hline 1283 & 2.4754 & 0.1259 & -0.01789 \\
\hline 1339 & 2.3557 & 0.1232 & -0.00084 \\
\hline 1394 & 2.2684 & 0.1217 & -0.02663 \\
\hline 1450 & 2.1176 & 0.1040 & -0.03149 \\
\hline
\end{tabular}
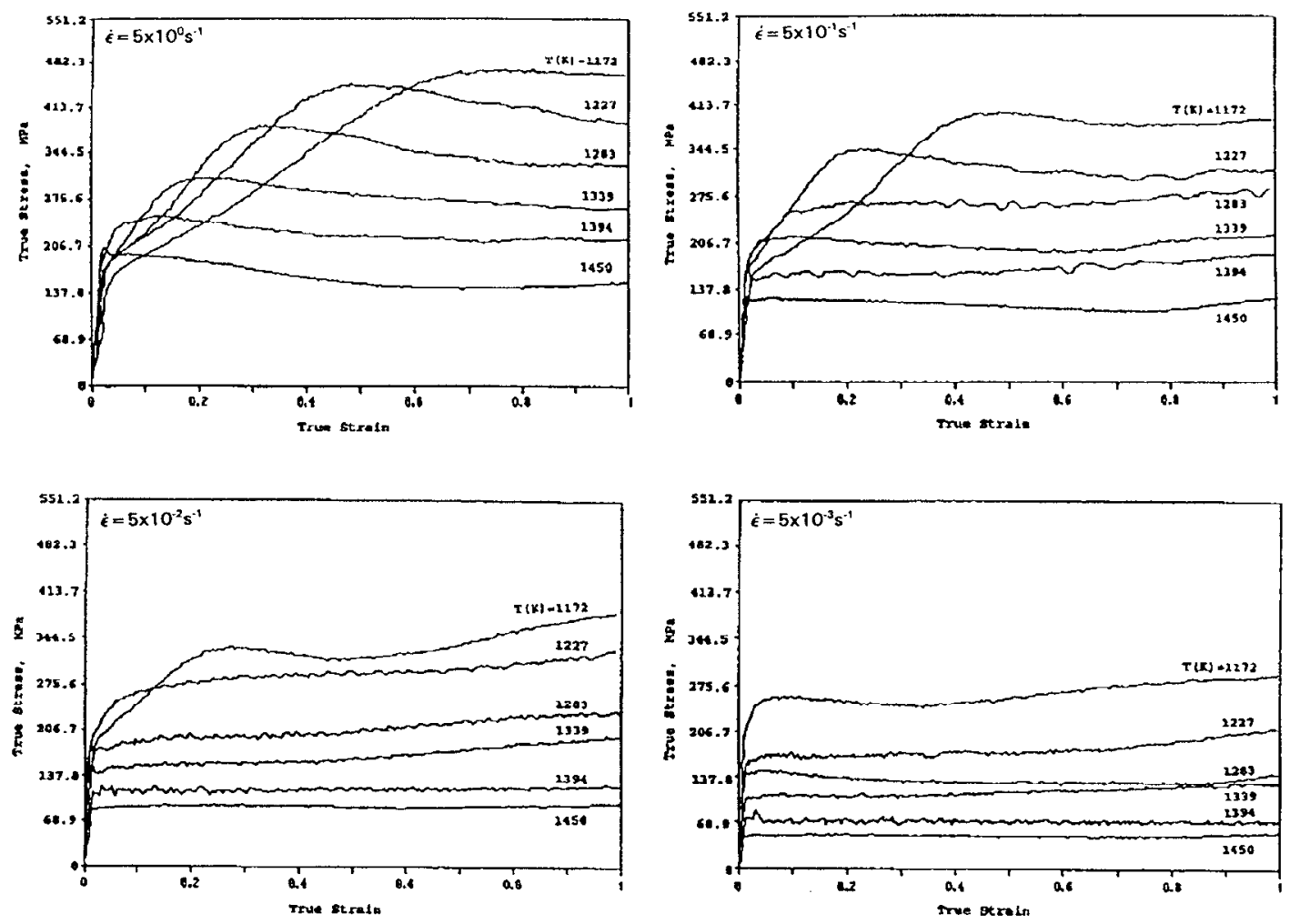

Figure 1. True stress-true strain flow curves for Alloy 718 deformed at 900 to $1177^{\circ} \mathrm{C}(1173$ to $1450 \mathrm{~K}$ ) for true strain rates of 0.005 to $5 \mathrm{sec}^{-1}$. 


\section{Apparent Activation Energy}

The apparent activation energy, Q, was obtained in the usual way. Substituting Eq.(4) into Eq.(3), we obtain:

$$
\dot{\epsilon}=\operatorname{Af}(\sigma) \exp (-\mathrm{Q} / \mathrm{RT})
$$

Taking $\log$ in both sides of Eq.(6), we obtain:

$$
\ln \dot{\epsilon}=\ln [\operatorname{Af}(\sigma)]-\mathrm{Q} / \mathrm{RT}
$$

and

$$
\log \dot{\epsilon} / \log (\mathrm{e})=\ln [\mathrm{Af}(\sigma)]-\mathrm{Q} / \mathrm{RT}
$$

so

$$
\log \dot{\epsilon}=\log (\mathrm{e})\left\{\ln [\mathrm{Af}(\sigma)]-\mathrm{Q} / \mathrm{RT}^{*} \log (\mathrm{e})\right.
$$

For a given $\sigma$, we can plot $\log \dot{e}$ versus $1 / \mathrm{T}$ as shown in Figure 2. From this figure, the slope, $k$, of the curves $\log (\dot{\epsilon})-1 / T$ is the quantity of $-Q / R^{*} \log (e)$, i.e.

$$
\mathrm{k}=-\mathrm{Q} / \mathrm{R} * \log (\mathrm{e})
$$

and

$$
\mathrm{Q}=-\mathrm{kR} / \log (\mathrm{e})
$$

The values of the calculated slope, $\mathrm{k}$, at various $\sigma$ values is presented in Table 2 . The results from this table indicate that values of $k$ are very similar. The average value of $\mathrm{k}$ for a strain of 0.6 is $-0.2536 \times 10^{4}$, hence, the corresponding apparent activation energy $\mathrm{Q}$ is $485 \mathrm{KJ} / \mathrm{mole}$. This value is higher than the $423 \mathrm{KJ} /$ mole value determined by Weis et $\mathrm{al}^{(7)}$ to describe the peak flow stress data obtained from comparable size 718 ingot product. However, both values are within the range of $Q$ values reported for nickel base superalloys.

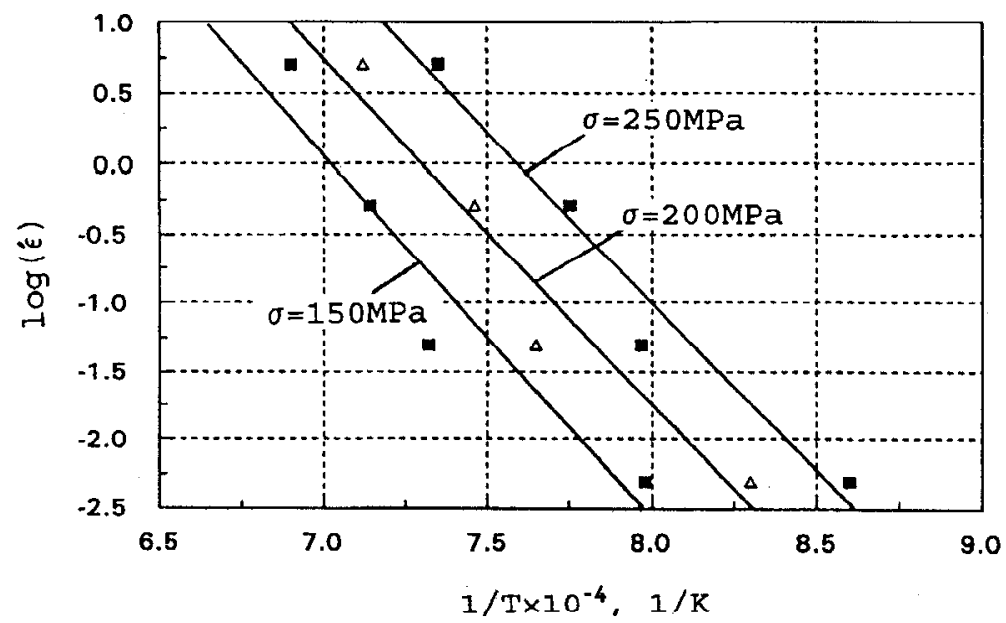

Figure 2. Plot $\log (\dot{\epsilon})$ vs $1 / \mathrm{T}$ at different stresses $(\epsilon=0.6)$ 
Table 2. Apparent Activation Energy Q for Different Experimental Conditions

\begin{tabular}{|c|c|c|c|}
\hline Stress, MPa & Strain & $\mathbf{k}, \mathbf{x 1 0 ^ { 4 }}$ & $\mathbf{Q}, \mathbf{K J} / \mathbf{m o l}$ \\
\hline \hline 250 & 0.6 & -2.4265 & 464.5 \\
\hline 200 & 0.6 & -2.5262 & 483.6 \\
\hline 150 & 0.6 & -2.6573 & 508.7 \\
\hline
\end{tabular}

Using the calculated activation energy, we can then calculate the Zener-Hollomon parameters $\mathrm{Z}=\dot{\epsilon} \exp (\mathrm{Q} / \mathrm{RT})$, the logarithm of this expression should be in linear relation with the peak-stress, $\sigma_{\mathrm{p}}$, see Figure 3 .

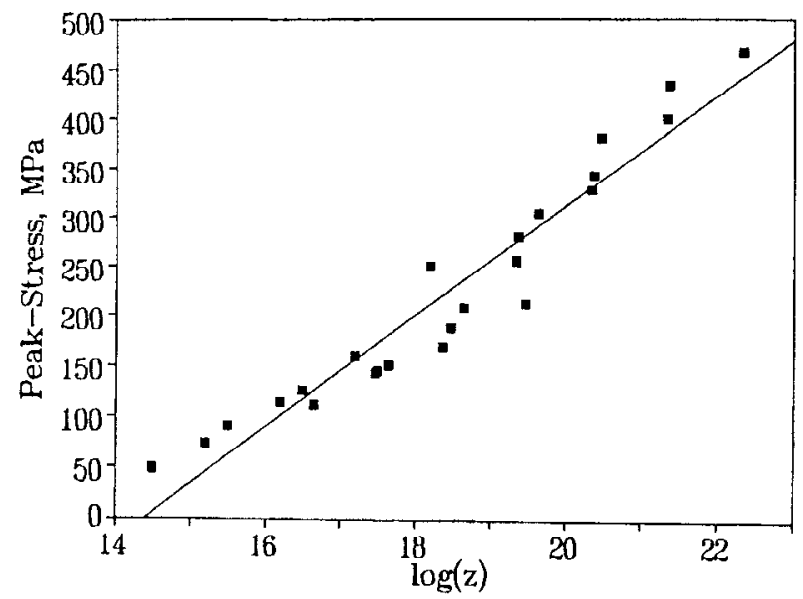

Figure 3. Relation between the peak-stress $\left(\sigma_{\mathrm{p}}\right)$ vs $\log (\mathrm{Z}) .(\mathrm{Q}=485 \mathrm{KJ} / \mathrm{mol}, \mathrm{T}=1172$ to $1450 \mathrm{~K}, \dot{\epsilon}=0.005$ to $5 \mathrm{~s}^{-1}$, and $\epsilon=0.6$ ).

\section{Strain Rate Sensitivity}

It is well understood that the high temperature flow stress behavior of a given material is strongly dependent on the deformation temperature and strain rate. Numerous studies have suggested different mathematical relations in an attempt to correlate the flow stress obtained at constant temperature and strain rate. The constant temperature flow stress equation is usually written as

$$
\sigma=\mathrm{K} \dot{\epsilon}^{\mathrm{m}}
$$

where $\mathrm{K}$ is a temperature-dependent strength parameter, and $\mathrm{m}$ is the strain rate sensitivity parameter. For pure metals, $m$, may be constant at different strain rates, but for complex alloys, $m$ will vary with temperature and strain rate.

Taking $\log$ in both sides of Eq.(11), we obtain

$$
\log \sigma=\log \mathrm{K}+\mathrm{m} \log \dot{\epsilon}
$$


and

$$
\mathrm{m}=\partial(\log \sigma) / \partial(\log \dot{\epsilon})
$$

or from Eq.(5) and using the constants calculated in Table 1 we have

$$
\mathrm{m}=\mathrm{b}+2 * \mathrm{c} * \log \dot{c}
$$

Figure 4 shows the three dimensional distribution of $\mathrm{m}$ as a function of $\log \dot{\epsilon}$ and $\mathrm{T}$, and Figure 5 shows the contour map of $\mathrm{m}$ with respect to $\log \dot{\epsilon}$ and $\mathrm{T}$. It is evident that in Alloy $718, \mathrm{~m}$ varies with strain rate and temperature. In general, the results from Figures 4 and 5 indicate that the strain rate sensitivity parameter $(\mathrm{m})$ decreases as the strain rate increases and the deformation temperature decreases.

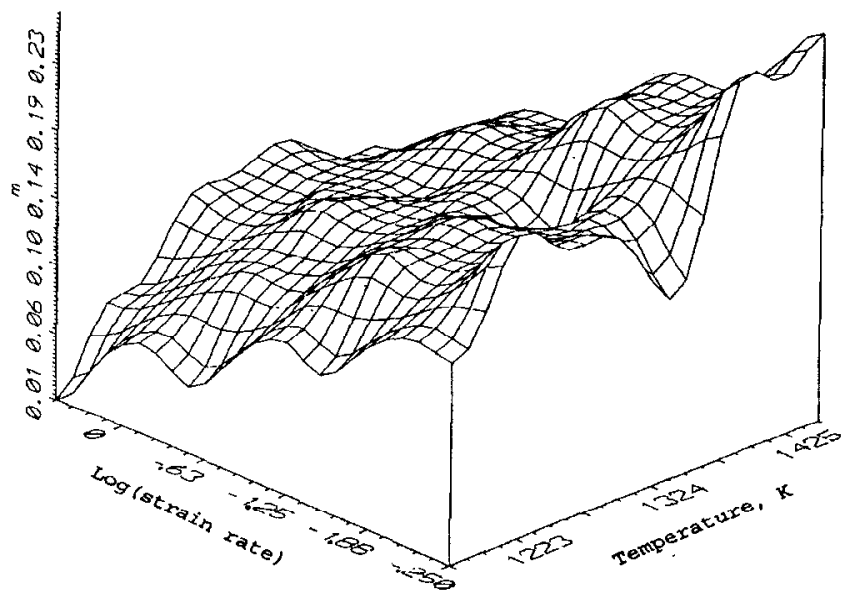

Figure 4. Three-dimensional distribution map of strain rate sensitivity parameter $\mathrm{m}$ with strain rate and temperature at 0.6 strain.

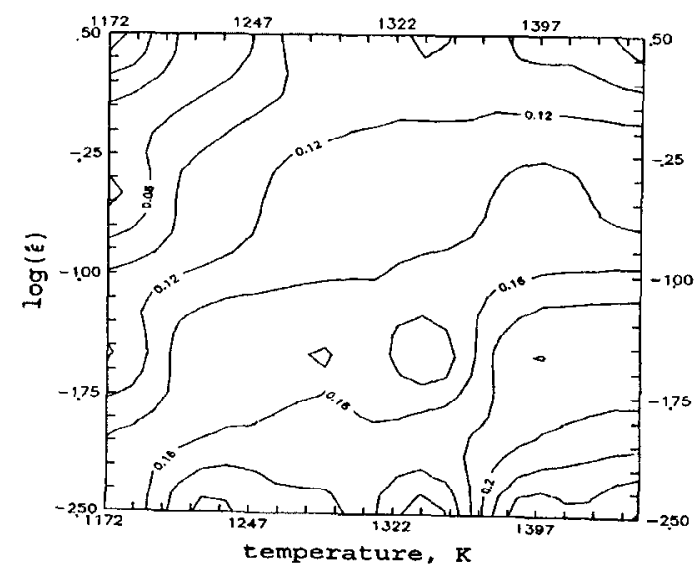

Figure 5. Contour map of strain rate parameter $\mathrm{m}$ with strain rate and temperature. 


\section{Hot Deformation Map}

The constitutive behavior of Alloy 718 at high temperature over a range of strain rates can be analyzed with the aid of the recently developed Dynamics Material Model ${ }^{[4-6]}$ According to Gegel and Prasad when a workpiece is deformed at a given temperature and strain, the power dissipation characteristics of the material can be attributed to microstructural changes. These changes can be evaluated through strain rate sensitivity maps obtained as a function of temperature and strain rate. ${ }^{[4,5]}$ However, it is important to note that although these processing maps can be interpreted in terms of specific microstructural mechanisms which take place at a given temperature and strain rate regime, the validity of the information provided by the maps should be substantiated by proper microstructural analysis.

The typical constitutive relation for a simple dissipator is schematically represented in Figure 6. ${ }^{[5]}$
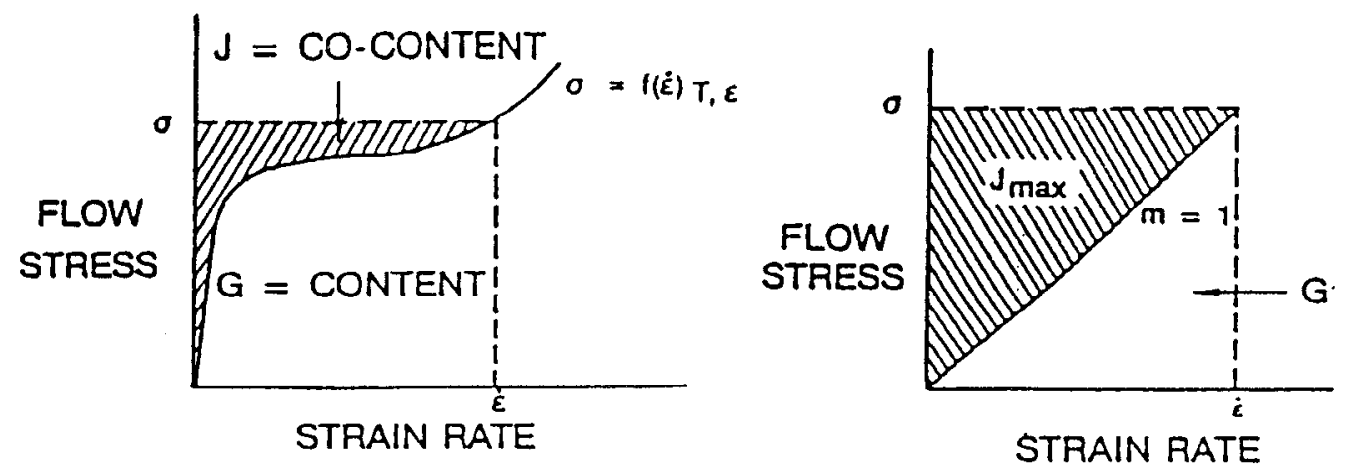

Figure 6. Constitutive relation in high temperature deformation.

When hot deformation takes place the instantaneous power absorbed by the material is the product of the flow stress and the strain rate $(\sigma \hat{\epsilon})$. This instantaneous power can be written as:

$$
\sigma \dot{\epsilon}=\int_{0}^{\dot{\epsilon}} \dot{\epsilon} \sigma \mathrm{d} \dot{\epsilon}+\int_{0}^{\sigma} \dot{\epsilon} \mathrm{d} \sigma
$$

or

$$
P=G+J
$$

where

$$
\begin{aligned}
& \mathrm{G}=\int_{0}^{\dot{\epsilon}} \sigma \mathrm{d} \dot{\epsilon} \\
& \mathrm{J}=\int_{0}{ }_{0} \dot{\epsilon} \mathrm{d} \sigma
\end{aligned}
$$

$\mathrm{G}$ is the dissipator content (area below curve) and $\mathrm{J}$ is the dissipator co-content (area above the curve), see Figure 6. 
Substituting Eq.(11) into Eq.(17), (18), and integrating them, the following expression can be obtained

$$
\begin{gathered}
\mathrm{G}=\int_{0}^{\dot{\epsilon}} \sigma \mathrm{d} \dot{\epsilon}=\int_{0}^{\sigma}(\sigma / \mathrm{A})^{1 / \mathrm{m}} \mathrm{d} \sigma=\dot{\epsilon} \sigma \mathrm{m} /(\mathrm{m}+1) \\
\mathrm{J}=\int_{0}^{\sigma} \dot{\epsilon} \mathrm{d} \sigma=\int_{0} \dot{ } \mathrm{A}^{\mathrm{m}} / \mathrm{d} \dot{\epsilon}=\dot{\epsilon} \sigma /(\mathrm{m}+1)
\end{gathered}
$$

rearranging and adding $\mathrm{J}$ and $\mathrm{G}$ yields

$$
\sigma \dot{\epsilon}=\dot{\epsilon} \sigma /(\mathrm{m}+1)+\dot{\epsilon} \sigma \mathrm{m} /(\mathrm{m}+1)
$$

the physical meaning of the expression shown in equation 21 indicates that the strain rate sensitivity ( $m$ ) can be regarded as a power-partitioning index which disperses energy between the energy dissipated by plastic deformation $(G)$ and the energy dissipated by the metallurgical events which occur dynamically (J). When is $\mathrm{m}<1$, the curve for $\sigma$ versus $\dot{\epsilon}$ appears similar to that shown in Figure $6 \mathrm{a}$. When $m=1$, the curve becomes a straight line, yielding the maximum dissipator co-content of area $\mathrm{J}_{\max }=\sigma \dot{\epsilon} / 2$, as shown in Fig. $6 \mathrm{~b}$.

A parameter $\eta$ can be defined using the ratio $\mathrm{J} / \mathrm{J}_{\max }$

$$
\eta=\mathrm{J} / \mathrm{J}_{\max }=2 \mathrm{~m} /(\mathrm{m}+1)
$$

where $\eta$ is a dimensionless parameter which describes how efficiently the material is dissipating energy by microstructural changes relative to $\mathrm{J}_{\max }$. The parameter $\eta$ can also be used to delineate stable and unstable regions. The range of $\mathrm{m}$ for stable material flow is given by

$$
0<m \leq 1
$$

This relationship can also be seen from Eq. (20), for $\eta$ to be between 0 to $100 \%$. The analysis discussed above provides hot deformation parameters to maximize the dynamic softening of a given material. That is, thermomechanical processing should be conducted in the regions with the highest value of $J$, which means the highest efficiency value. This is consistent with the intent of maximizing the $J$ co-content since this portion of the power directly contributes to dynamic metallurgical changes within a workpiece.

A three-dimensional processing map and its contour showing the variation of efficiency $\eta$ with $\mathrm{T}$ and $\dot{\epsilon}$ at an effective strain $\epsilon$ of 0.5 for Alloy 718 was constructed and is shown in Figures 7 and 8 . The map can be used to measure the dispersal of energy through metallurgical process with deformation temperature and strain rate. The hot deformation map presented in Figure 7 , is in good agreement with a previously reported recrystallization - temperature - time map for Alloy 718, see Figure 9.(1)

In summary, the hot deformation map developed for Alloy 718 using the Dynamic Materials Model defines the hot deformation conditions under which microstructural refinement can occur through dynamic recrystallization. However, the model does not clearly define the partial and/or non-recrystallized regimes. Significant advances in the understanding of microstructural refinement through thermomechanical processing have shown different ways to produce a well conditioned microstructure during hot deformation. Therefore, the combined use of hot deformation maps using the Dynamic Materials Model and Physical Model (recrystallization - deformation - temperature and/or recrystallization - temperature - 


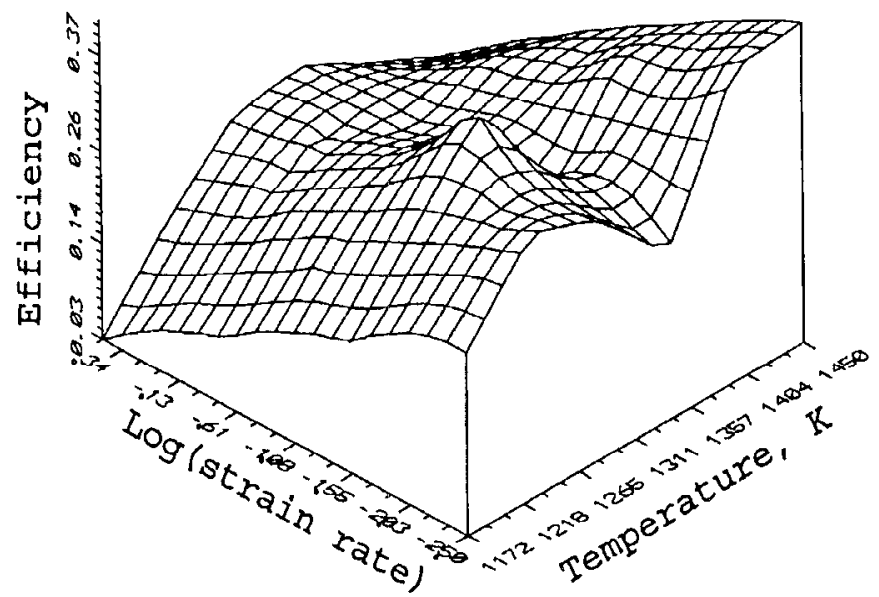

Figure 7. Threc-dimensional power dissipation map for Alloy 718 at a strain of 0.5 .

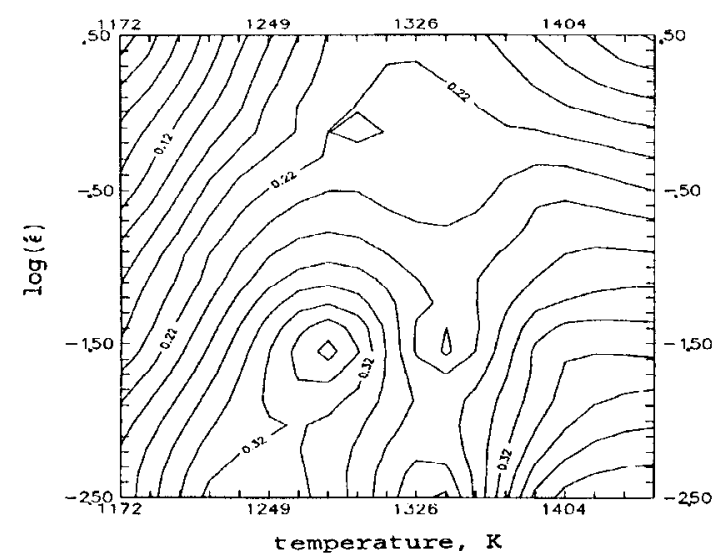

Figure 8. Constant efficiency contours of hot deformation processing map for Alloy 718.

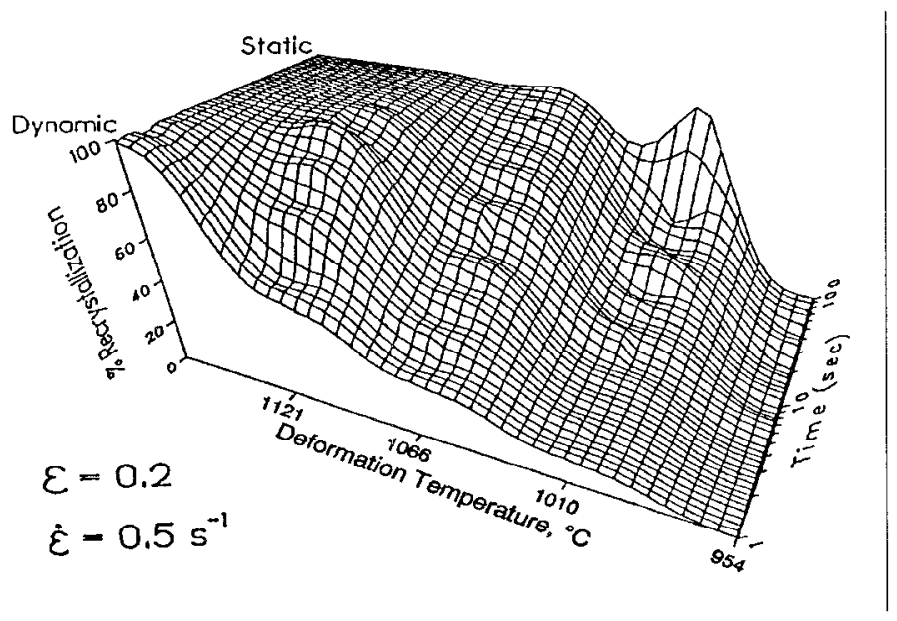

Figure 9. Recrystallization - Temperature - Time surface map for Alloy 718 under dynamic and static conditions. 
time) should provide a powerful tool to the understanding of microstructural optimization through thermomechanical processing.

\section{CONCLUSIONS}

1. Constitutive equations for Alloy 718 were derived to describe its hot deformation behavior.

2. The apparent activation energy $Q$ for Alloy 718 was determined to be about $483 \mathrm{KJ} / \mathrm{mol}$.

3. The strain rate sensitivity of Alloy 718 was determined.

4. The processing map for alloy 718 using the Dynamic Materials Model was determined. This map provides information under dynamic recrystallization takes place. These results are in good agreement with physical model maps published in the literature.

\section{ACKNOWLEDGEMENTS}

The authors wish to acknowledge the financial support from the Material Research Center

of the University of Pittsburgh.

\section{REFERENCES}

1. C.I. Garcia, D.E. Camus, E.A. Loria and A.J. De Ardo, "Microstructural Refinement of As Cast 718 via Thermomechanical Processing", Proceedings of The International Symposium on Superalloys 718, 625 and Various Derivatives, E.A. Loria, ed., (TMS 1991, pp.147-160.

2. C.I. Garcia, A.K. Lis, E.A. Loria and A.J. De Ardo, "Thermomechanical Processing and Continuous Cooling Transformation Behavior of IN-718", in Superalloys 1992 International Symposium Proceedings, S.D. Antolovich et al Ed., TMS, 1992, pp. $527-$ 536.

3. J.M. Alexander, "Mapping Dynamic Material Behavior", in Modelling Hot Deformation of Steels, J.G. Lenard Ed., Springer-Verlag, 1989, pp.101-115.

4. H.L. Gegel, "Synthesis of Atomistic and Continuum Modelling to describe Microstructure, in Computer Simulation of Materials Science, Ed. by R.J. Arsenault et al, ASM, 1988, pp.281-344.

5. T.D.R.K. Prasad, et al, Metall. Trans. A, Vol.15A, Oct. 1984, pp.1883-1892.

6. J.F. Thomas, et al, "Constitutive Equation for High-Temperature Deformation", in Computer Simulation for Materials Science, Ed. by R.J. Arsenault et al, ASM, 1988, pp. 279-290.

7. W.J. Weis, et al, "The Hot Deformation Behavior of an As-Cast Alloy 718 Ingot", Superalloy 718-Metallurgy and Applications, E.A. Loria Ed., TMS, 1989, pp.135-154. 